Article

\title{
Binding mode knowledge of new possible anti- hypertensive compounds designed in silico using Neutral Endopeptidase (NEP) as a target.
}

\author{
Emilio Lamazares ${ }^{1}$, Yudith Cañizares-Carmenate ${ }^{2}$, Juan A. Castillo-Garit ${ }^{2,3}$ and Karel Mena- \\ Ulecia $4,5, *$ \\ 1 Universidad de Concepción, Biotechnology and Biopharmaceutical Laboratory, Pathophysiology \\ Department; School of Biological Sciences, Concepción, Chile; elamazares@udec.cl \\ 2 Unit of Computer-Aided Molecular "Biosilico" Discovery and Bioinformatic Research (CAMD-BIR Unit), \\ Facultad de Química-Farmacia, Universidad Central "Marta Abreu" de Las Villas, Santa Clara, Villa Clara, \\ Cuba; yudithc@uclv.edu.cu \\ 3 Unidad de Toxicología Experimental, Universidad de Ciencias Médicas "Dr. Serafín Ruíz de Zárate Ruíz; \\ jacgarit@yahoo.es; juan.castillo@uv.es \\ 4 Departamento de Ciencias Biológicas y Químicas, Facultad de Recursos Naturales. Universidad Católica \\ de Temuco. Ave. Rudecindo Ortega 02950, Temuco, Chile; kmena@uct.cl \\ 5 Núcleo de Investigación en Bioproductos y Materiales Avanzados (BIOMA), Facultad de Ingeniería, \\ Universidad Católica de Temuco. Ave. Rudecindo Ortega 02950, Temuco, Chile.; kmena@uct.cl
}

* Correspondence: kmena@uce.cl; Tel.: +56-9-6603-2531 (C.L.)

\begin{abstract}
Arterial hypertension is a health problem that affects millions of people around the world. Particularly in Chile, according to the last health survey in 2019, 28.7\% of the population had this condition, and arterial hypertension complications cause one in three deaths per year. In this work, we have used molecular simulation tools to evaluate new compounds designed in silico by our group as possible anti-hypertensive agents, taking Neutral Endopeptidase (NEP) as a target, a key enzyme in the arterial hypertension regulation at the level kidney. We use docking experiments, molecular dynamics simulation, free energy decomposition calculations (by MM-PBSA method), and ligand efficiency analysis to identify the best anti-hypertensive agent pharmacokinetic and toxicological predictions (ADME-Tox). The energetic components that contribute to the complexes stability are the electrostatic and Van der Waals components; however, when the ADME-Tox properties were analyzed, we conclude that the best anti-hypertensive candidate agents are Lig783 and Lig3444, taking Neutra Endopeptidase as a target.
\end{abstract}

Keywords: Neprilisin; Docking; Molecular Dynamics Simulation; MM-PBSA; LIgand Efficiency Metrics; ADME-Tox

\section{Introduction}

Arterial hypertension is a health problem that affects millions of people around the world. The World Health Organization (WHO) has stated that more than 9.8 million people worldwide die every year from the arterial hypertension consequences (1). Particularly in Chile, according to the last health survey in $2019,28.7 \%$ of the population had this condition, and one in three deaths per year is caused by arterial hypertension complications (2). For this reason, it is interesting to increase the search for more effective drugs to fight this disease.

One of the essential pharmacological targets that have mostly focused on current research is the renin-angiotensin-aldosterone system (RAAS)(3). This system is the primary regulator of fluid and ion balance at the kidney level(4). The RAAS comprises a series of vital proteins in the arterial hypertension regulation in the kidney. One of these enzymes is the Neutral Endopeptidase, also 
called Neprilysin (NEP)(5-7). This protein is responsible for the degradation of natriuretic peptides, degradation of kinins, and adrenomedullin $(8,9)$. The natriuretic peptides can be seen as endogenous inhibitors of the renin-angiotensin system (RAS)(10).

NEP is one of the enzymes with more significant pharmacological potential in cardiovascular, inflammatory diseases, and arterial hypertension treatment in general. The NEP inhibition increases the bradykinin and adrenomodulin levels. Therefore inhibiting vasopeptidases, reducing vasoconstriction, enhancing vasodilation, and improving sodium balance and water. Also, decreasing peripheral vascular resistance and blood pressure while improving local blood flow. Inside the blood vessel walls produce a reduction of vasoconstriction and proliferative mediators such as angiotensin II. Further increased local bradykinin levels (and, in turn, nitric oxide) and natriuretic peptides $(7,11-13)$.

Currently, there are several NEP inhibitors drugs on the market, such as candoxatril and its successor Ecadotril (14). However, these synthetic drugs have specific side effects such as cough, taste disturbances, rashes, or angioneurotic edema $(15,16)$. Because of the arterial hypertension pathological complexity, it is necessary to research, design, and development new compounds to find more safe, innovative, and economic NEP inhibitors for the prevention and remedy of hypertension (17). For this reason, in previous works, we have computationally designed new ligands as possible anti-hypertensive agents, based on the QSAR-IN methodology (18), taking Thermolysin as the target, which is a protein of the M4 family and presents structural similarities with NEP (19-22). That is why we have performed a set of computational experiments based on molecular mechanics (Docking, Molecular Dynamics Simulation, MM-PBSA, Ligand Efficiency Metrics, and AMDE-Tox calculations) to analyze the compounds. Finally, the anti-hypertensive drugs best candidates based on NEP as a target will be obtained.

\section{Results and Discussion}

In the previous section, we have obtained five molecules as possible anti-hypertensive agents (18). The structure of these molecules is represented in Figure 1. These ligands were subjected to docking experiments with Neprilysin to see how these compounds were oriented in this protein's active center.

\subsection{Docking experiments.}

The docking method can predict the binding mode at the pocket of a target protein (23-25). We test the docking experiment's reproducibility by redocking our HAO reference ligand (LigHAO). The LigHAO was obtained from the Neutral Endopeptidase crystallographic structure (26). The RMSD values were quantified for all ligands.

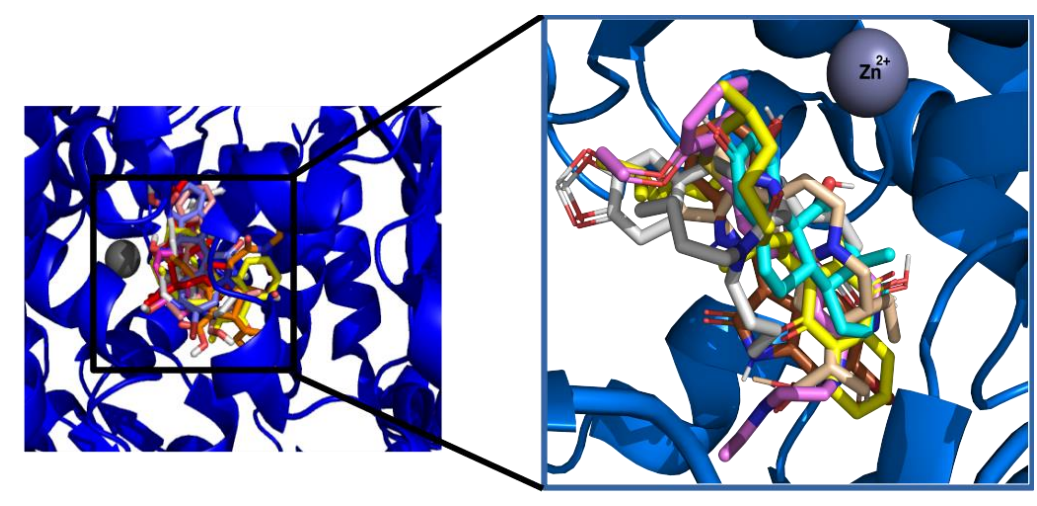

Figure 1. Alignment of all docked ligands in complex with Neutral Endopeptidase. 
As shown in Figure 1, all the ligands were oriented suitably in the NEP's active center, indicating that our docking experiment acceptably reproduces the crystallographic structure obtained from the Protein Data Bank (26). In addition to the visual analysis, we quantified the RMSD for each ligand, taking the LigHAO as a reference. As we can see in Table 1, 71.5\% of the poses analyzed ( 250 for each compound studied) had RMSD values lower than $2 \AA$. The RMSD value of $2 \AA$ was taken as a reference for a correct or incorrect (> $2 \AA$ ) docking resolution $(27,28)$. Only the Lig2177 compound had RMSD values greater than $2 \AA$.

Table 1. Parameters calculated from docking experiments for all complexes studied.

\begin{tabular}{lcccccc}
\hline Complexes & Cluster & $\begin{array}{c}\text { Total } \\
\text { Elements }\end{array}$ & $\begin{array}{c}\text { Full Fitness } \\
\text { (kcal/mol) }\end{array}$ & $\begin{array}{c}\Delta \mathrm{G} \text { binding } \\
(\mathbf{k c a l} / \mathbf{m o l})\end{array}$ & $\begin{array}{c}\text { RMSD } \\
(\AA)\end{array}$ & $\begin{array}{c}\text { Number } \\
\text { H-bond }\end{array}$ \\
\hline LigHAO-2YB91 & $50 / 50$ & $255 / 255$ & $-3184,06$ & $-20,86$ & 0,34 & 2 \\
Lig783-2YB9 & $36 / 37$ & $255 / 255$ & $-3635,44$ & $-7,12$ & 0,97 & 1 \\
Lig1022-2YB9 & $35 / 36$ & $254 / 255$ & $-3656,55$ & $-9,74$ & 0,70 & 0 \\
Lig2177-2YB9 & $39 / 39$ & $255 / 255$ & $-3583,70$ & $-8,41$ & 2,03 & 4 \\
Lig3444-2YB9 & $40 / 40$ & $255 / 255$ & $-3599,01$ & $-8,34$ & 0,37 & 0 \\
Lig6199-2YB9 & $44 / 44$ & $250 / 255$ & $-3652,27$ & $-10,11$ & 1,70 & 0 \\
\hline
\end{tabular}

${ }^{1}$ Ligand of reference from Protein Data Bank $(26,29)$

Base on the docking results presented in Table 1, all the complexes studied had binding energies above $7 \mathrm{kcal} / \mathrm{mol}$. The most negative $\Delta$ Gbinding was obtained in our reference ligand LigHAO (-20.86 kcal/mol). This compound was oriented in the NEP active center so that the carboxyl group formed by the oxygens $\mathrm{O} 2$ and $\mathrm{O} 3$ presented several non-covalent interactions. For example, the oxygen $\mathrm{O} 2$ of this functional group shows a negative charge density interacting electrostatically with the $\mathrm{Zn}^{2+}$ of NEP (2.09 $\AA$ ). Besides, oxygen 03 exhibits hydrogen bond (Hbond) interactions with the NH1 and NH2 groups of Arg717.

Furthermore, the other carboxyl group of LigHAO presents oxygen (OTX) that also interacts with the $\mathrm{Zn}^{2+}$ of NEP, stabilizing this complex. These interactions are also found in the Neutral Endopeptidase's crystallographic structure obtained from the Protein Data Bank (PDB id: 2YB9). These results confirm that our docking experiments had excellent reproducibility (Figure 2A).

The second most negative binding energy was found in the complex formed by Lig61992YB9 with a $\Delta$ Ginding value of $-10.11 \mathrm{kcal} / \mathrm{mol}$ (Table 1 ). This ligand was oriented in the NEP active center that the hydrophobic linear hydrocarbon skeleton was inserted into a hydrophobic pocket formed by the amino acids Arg102, Phe106, Asp107, and Tyr697, stabilizing this complex (Figure 2F).

Something similar happened with the Lig1022. This compound presented the third most negative binding energy $(-9.74 \mathrm{kcal} / \mathrm{mol})$. Like Lig6199, this ligand did not present H-bond interactions in the docking experiments because of its structural characteristic. It has a long apolar hydrophobic hydrocarbon chain, which is oriented in a hydrophobic pocket formed by the side chains of the amino acids Arg102, Phe106, Asp107, Thr708, Asp709, His711, and Arg717. This orientation confers excellent stability to the Lig1022-2YB9 complex (Figure 2D). 
The Lig2177 presented the fourth most negative binding energy of all the ligands studied. The Lig2177-2YB9 complex exhibited hydrogen bond interactions with Asn704 (3.15), Tyr697 (3.33), and with Asp709 (3.27 and 2.79). These interactions give this complex certain stability; hence its binding energy is $-8.41 \mathrm{kcal} / \mathrm{mol}$ (Figure $2 \mathrm{C}$ )

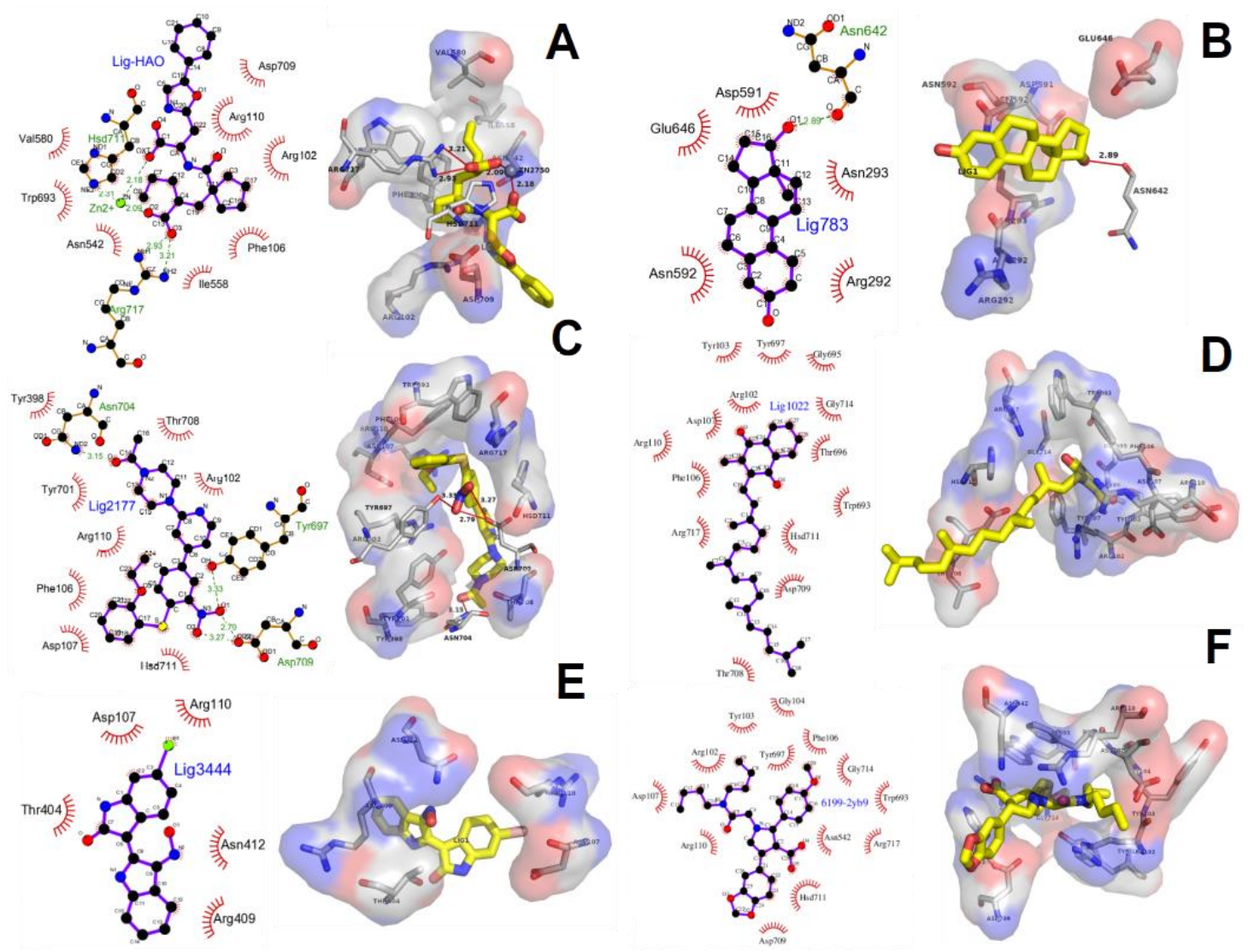

Figure 2. Graphical representation of the ligands binding modes designed in silico into the NEP Pocket: (A) HAO reference ligand, (B) Lig783, (C) Lig2177, (D) Lig11022, (E) Lig3444, and (F) Lig6199.

\subsection{Molecular Dynamics Simulation.}

To study the complexes' dynamic behavior, we have performed molecular dynamics simulations to know if the docking experiments' interactions are maintained during the $50 \mathrm{~ns}$ of simulation time. This method will also allow us to observe if these systems remain stable over time. As a stability criterion, we have quantified the RMSD, H-bond, RMSF, and Radius of gyration $(\mathrm{Rg})$ parameters, which are shown below.

\subsubsection{Root Means Squared Deviation (RMSD).}

We have obtained the RMSD parameter values during the $50 \mathrm{~ns}$ of molecular dynamics simulation as stability proof. As shown in Figure 3, all systems remained stable over time with RMSD values lower than $1.4 \AA$. It should be noted that all the systems studied remained stable after $8 \mathrm{~ns}$ of simulation time. The Lig2177-2YB9, with an RMSD value average of $0.919 \pm 0.057 \AA$ resulted in the most stable complex behavior. This complex presented the fourth most negative binding energy in docking experiments (out of six systems studied), which leads us to think that there are losses of interactions compared to the docking results.

The second most stable complex taking into account the RMSD values was Lig3444-2YB9 $(0.923 \pm 0.068 \AA)$. According to the molecular dynamics simulations performed, this system had 
similar behavior to Lig2177 in the docking results, reaffirming the previous approach about the loss of interactions in the docking experiments.

The less stable complex of all those studied (which does not mean that its behavior is unstable) was Lig6199-2YB9 with an RMSD average value of $1.10 \pm 0.105 \AA$, like the complex formed by our reference ligand and the NEP, which had the second-highest RMSD value of all $(1.063 \pm 0.079 \AA)$. Both systems had the two most negative energies in the docking results, indicating that the complexes' dynamic behavior differed from that found in the docking results. To find an explanation for this behavior, We will analyze the ligands' interactions designed in silico in the Neutral Endopeptidase active center at the molecular level.
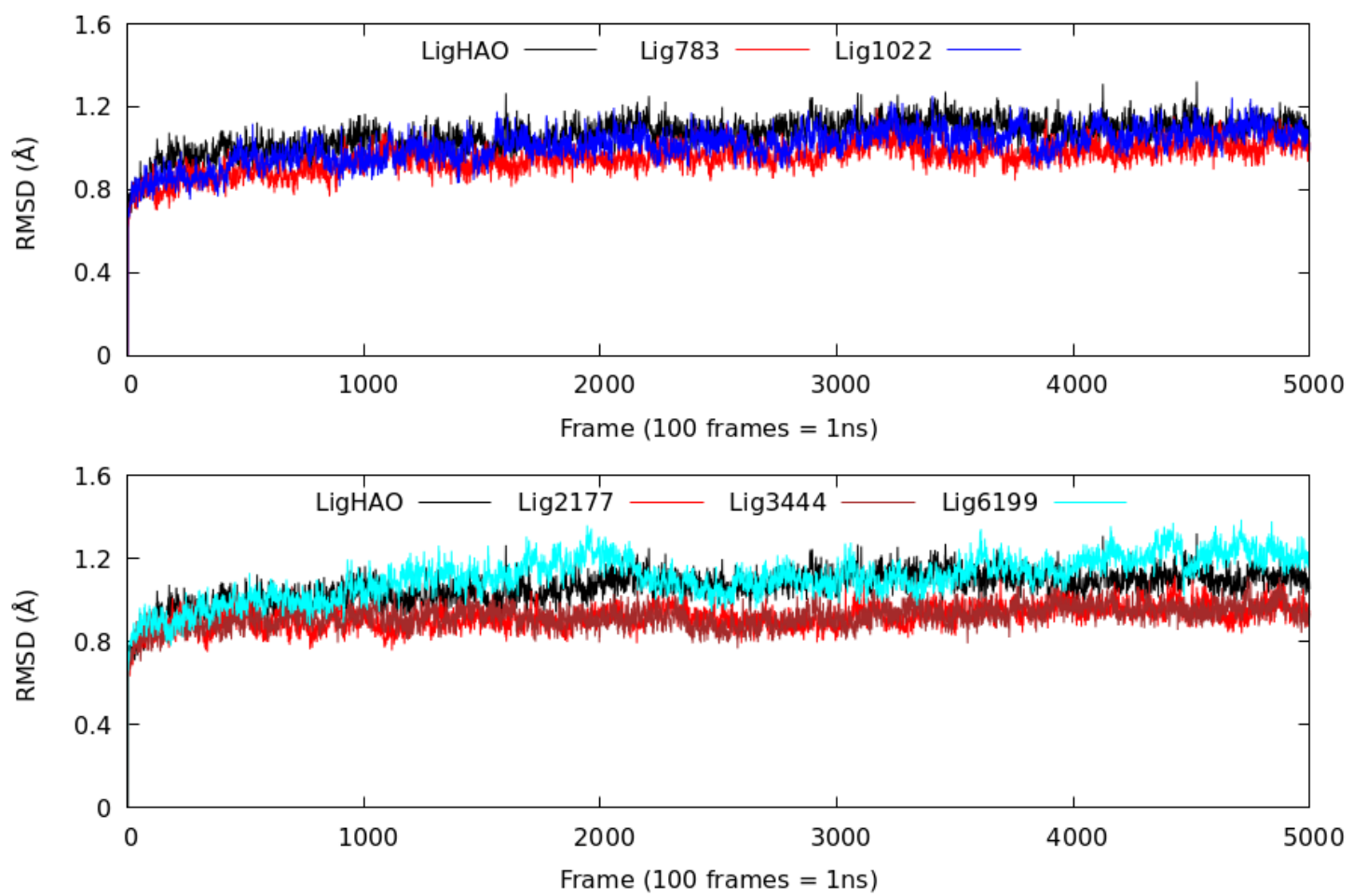

Figure 3. Plots of RMSD values against simulation time during $50 \mathrm{~ns}$ of molecular dynamics simulations of the studied complexes.

\subsubsection{Hydrogen Bond interactions (H-bond).}

To explain the molecular level of the behavior found in the RMSD parameter measurements, we have quantified the hydrogen bond (H-bond) amount and stability generated during the simulation time. Besides, we can verify if the docking experiments' interactions are preserved over time.

As shown in Figure 4, the complex formed by our reference ligand and NEP (LigHAO2YB9) was the complex with the most H-bond interactions with an average of $3.27 \pm 0.98$. From 3 ns, these interactions remained stable until the end of the simulation time. To corroborate this approach, we quantify the interactions found and observe that the hydrogen bonds formed by LigHAO-O1--HN-Arg110 and LigHAO-OX--HNArg102 had 100\% occupancies. This result means that the interactions were keeping by below $3 \mathrm{~A}$ during the 50 ns of simulation time, which was our occupation's cutoff parameter. These interactions are the ones that give the most stability to this system. 

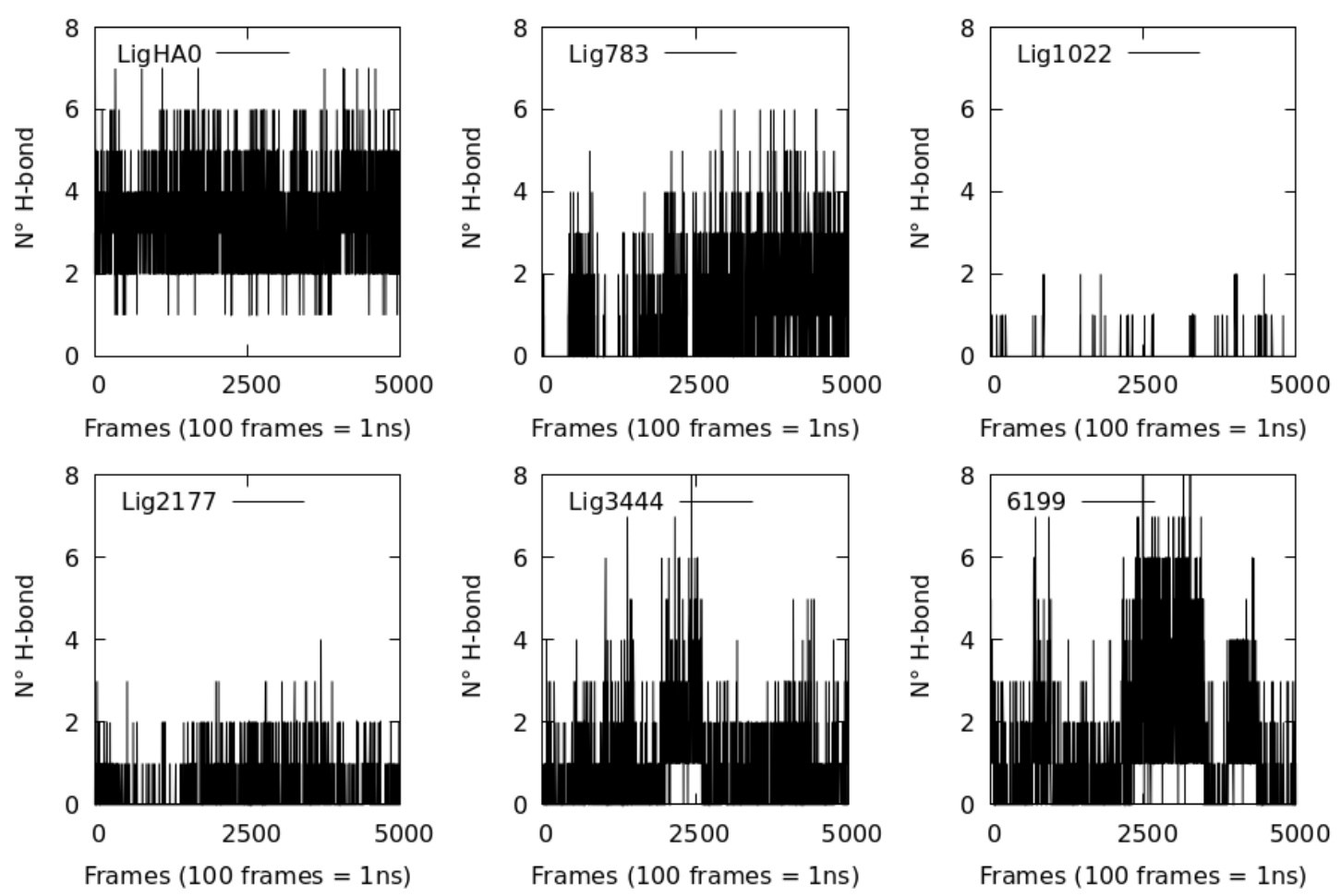

Figure 4. The H-bond number for all complexes studied during 50 ns of simulation time

The second complex with the highest hydrogen bond interaction numbers was Lig61992 YB9 (1.16 \pm 1.48$)$. This complex's dynamic behavior was unique since the $\mathrm{h}$-bonds remained stable during the first $25 \mathrm{~ns}$ of simulation time, reaching certain instability from nanosecond 26 to 45 . From there, it stabilized again until the end of the simulation. This behavior is reflected in the sampling standard deviation, which behaved well above the sample mean. This approach is reflected in the occupation of the hydrogen bridge interactions, were in none of the cases did they exceed $50 \%$ of this parameter. The highest occupations were found in the hydrogen bonds formed by Arg114-OE2--HO-Lig6199 (34\%) and Arg110-NH--O4-Lig6199 (32\%). The other complexes studied had an average number of hydrogen bridge interactions below one and with occupancies below $5 \%$, denoting certain instability in dynamic behavior.

LigHAO-2YB9 and Lig6199-2YB9 were the complexes with the most negative binding energies in the docking experiments, agrees with the results obtained in this section. However, the results obtained with the RMSD parameter do not agree with what is shown here. What is necessary to analyze other parameters extracted from the molecular dynamics simulations such as radius of gyration and RMSF, results that we will display below.

\subsubsection{Radius of Gyration (Rg).}

The results analyzed have given a certain degree of agreement between the docking experiments and the hydrogen bridges quantification obtained from the trajectories. However, there are discrepancies in the results obtained from the RMSD parameter, so we have to analyze the radius of gyration in the simulation time.

The $\mathrm{Rg}$ is defined as the mean square distance of the mass of a set of atoms with a common mass center (30-32). In other words, this parameter gives us an idea of the compaction degree of the complexes studied during the simulation time. With $\mathrm{Rg}$, we can compare the influence of ligands on the dynamic behavior of neutral endopeptidase. 

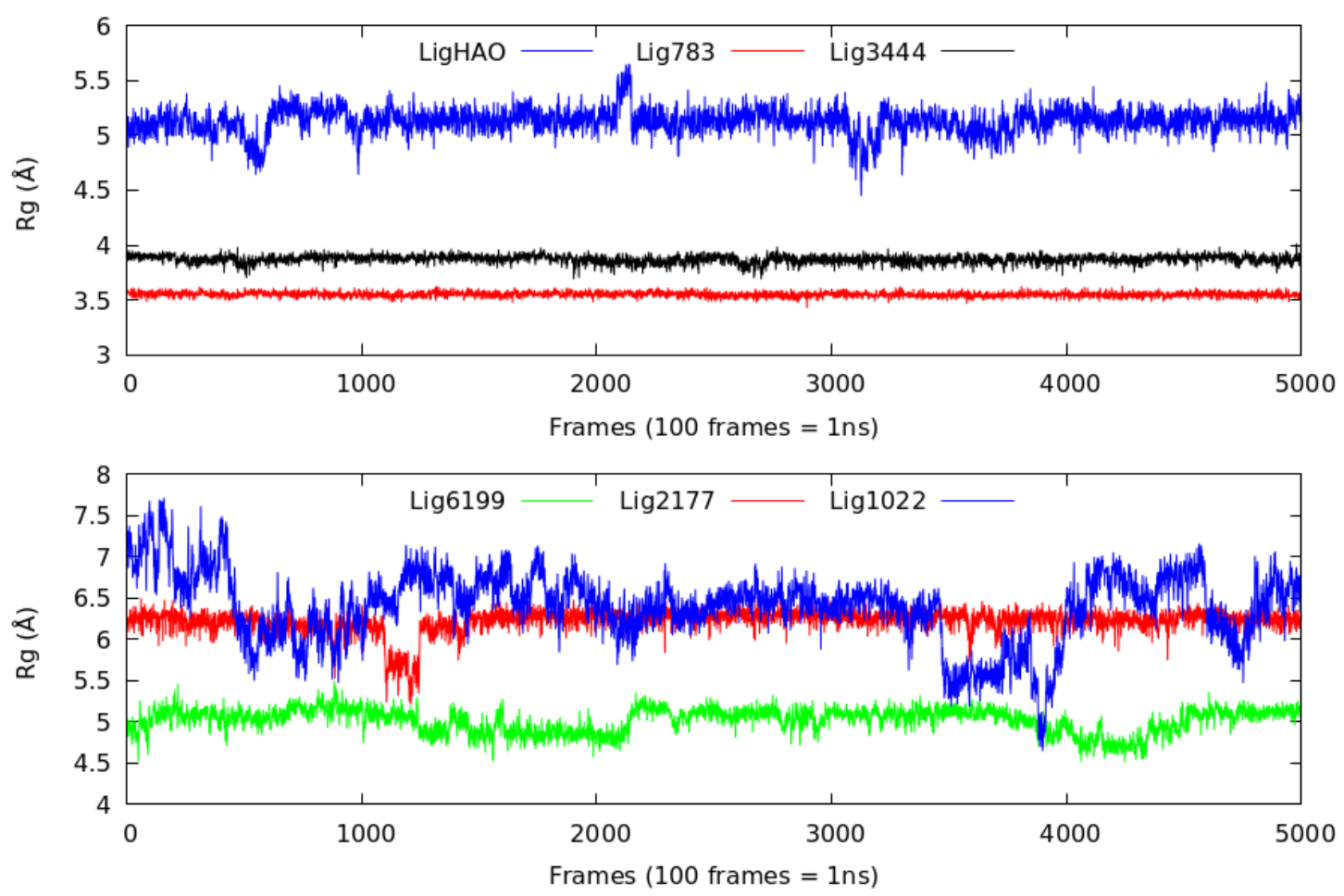

Figure 5. The Rg of the Lig-2YB9 complexes during $50 \mathrm{~ns}$ of simulation time at $298.15 \mathrm{~K}$.

As shown in Figure 5, all the systems studied had Rg values greater than 3.5 A, higher than other complexes consulted in the literature(30-32). However, it is necessary to explain that the complexes formed by Lig783-2YB9 and Lig3444 -2YB9 were the complexes with the lowest Rg fluctuation during the $50 \mathrm{~ns}$ of molecular dynamics. This result indicates that these systems were the most compact of all those studied. This behavior can be explained (at this point in the analysis) because both complexes had the lowest RMSD values (together with Lig2177-2YB9) and the lowest standard deviation values obtained from the MD simulations.

The system with the most significant fluctuation in the Rg parameter was the Lig1022-2YB9. This complex was the one with the least amount of hydrogen bond interactions (Figure 4), and it was also the complexes that had the lowest occupancy of these interactions. It should be noted that the highest $\mathrm{H}$-bond interactions occupancy found in this system was formed by Asn542NH--O-Lig1022 with $0.54 \%$, indicating the instability of this type of interactions, which could explain the Lig1022-2YB9 low degree compaction.

\subsubsection{Root Means Squared Fluctuation (RMSF).}

The RMSF parameter will help us understand the differences in flexibility between the amino acid residues at the molecular level that make up NEP's structure when ligands designed in silico are attached to the pocket. A high value of this parameter indicates high flexibility, which could be inferred in a greater degree of freedom of movement; however, low RMSF values indicate more restricted movements during the molecular dynamics simulation (33).

As shown in Figure 6, specific differences exist between the NEP backbone without ligands in the active center, and the complexes studied. This difference lies in the greater amino acid flexibility in the absence of ligands. However, we observed that the amino acid residues between 520 and 670 have the least freedom of movement in the backbone. Within this sequence are the amino acids that constitute the active center of NEP (Asn542, His583, Glu584, His587, and Glu646). 

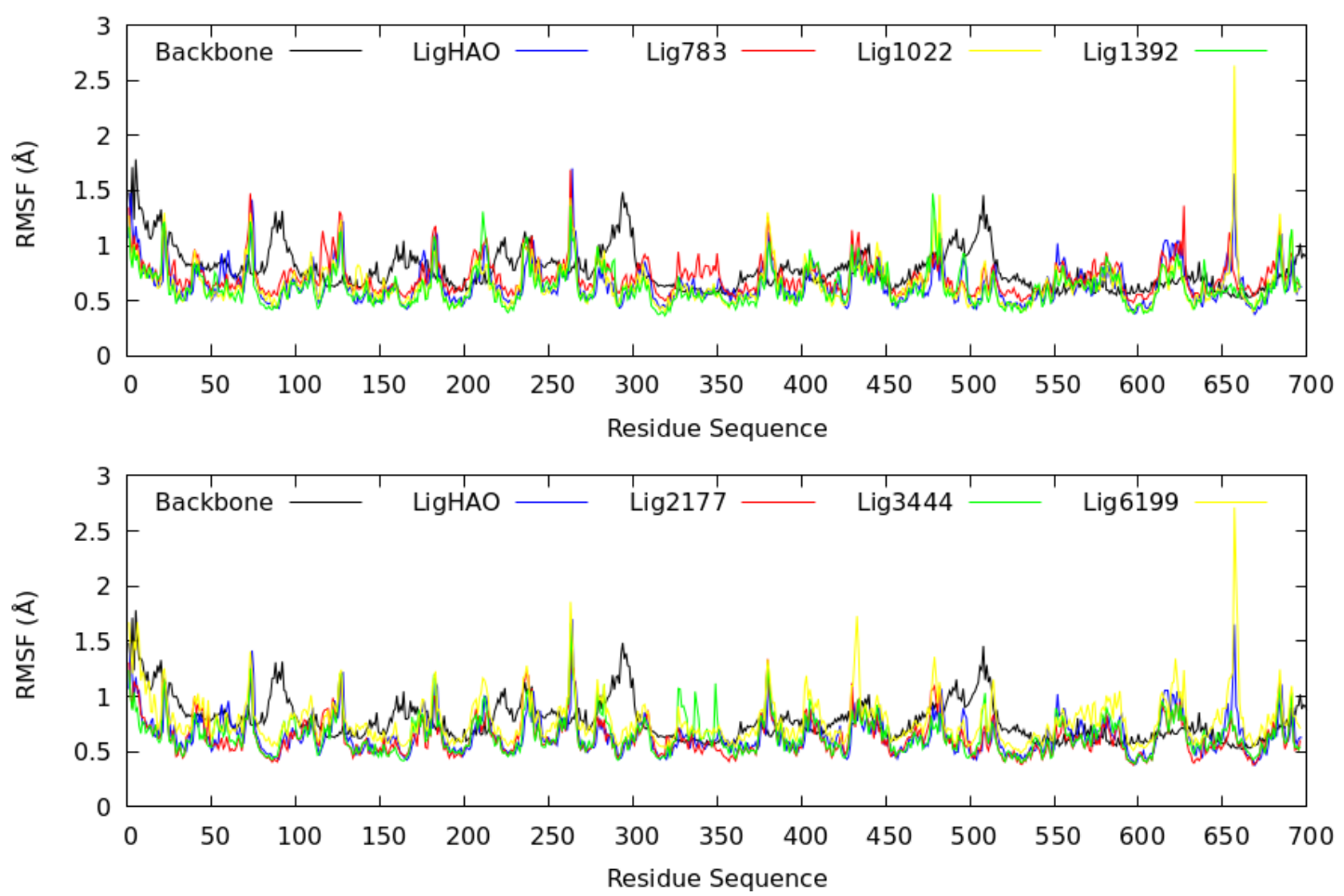

Figure 6. Root Means Squared Fluctuation (RMSF) behavior of the backbone and complexes during 50 ns of simulation time at 298,15 Kelvin.

The systems studied had lower RMSF values concerning the backbone, indicating that the ligands' binding with NEP limits the amino acids flexibility that makes up our target protein's active center. Of all the complexes, those formed by the ligands Lig1022 and Lig6199 had the highest RMSF values, indicating that these compounds in the NEP active center make the amino acids appear more flexible. This fact explains the Lig1022-2YPB complex behavior when the Rg was analyzed, which was the system that had the most fluctuation in this parameter, being the least compact complex of all those studied in this work.

\subsection{Molecular Mechanics- Poison-Boltzman Surface Area methods (MM-PBSA).}

To determine the energy factors that contribute to the stabilization or destabilization of the complexes studied, we have analyzed the decomposition of energy using the MM-PBSA method(34-38).

The most negative binding energy was obtained by the Lig3444-2YB9 complex ( $\Delta$ Gbinding $=$ $78.99 \pm 8.67 \mathrm{kcal} / \mathrm{mol}$ ) (Table 2). This system was the second with the lowest RMSD and the second with the highest hydrogen bond number obtained from molecular dynamics simulations. This complex has been the most stable of all those studied, considering a comprehensive analysis of the results obtained so far. Our results agree with previous works obtained with this same compound but using another M4 family metalloprotein similar to Neprilysin (24). According to $\Delta$ Gbinding, other stable complexes were Lig6199-2YB9, Lig10222YB9, and Lig2177-2YB9, making them good candidates anti-hypertensive drugs.

Table 2. Predicted binding free energies $(\mathrm{kcal} / \mathrm{mol})$ and individual energy terms calculated from molecular dynamics simulation through the MM-PBSA methodology.

\begin{tabular}{cccccc}
\hline Complexes & $\Delta \mathrm{G}_{\text {binding }}$ & $\Delta \mathrm{E}_{\text {elect }}$ & $\Delta \mathrm{E}_{\mathrm{vdw}}$ & $\Delta \mathrm{G}_{\text {Polar }}$ & $\Delta \mathrm{G}_{\text {Apolar }}$ \\
\hline
\end{tabular}




\begin{tabular}{lccccc}
\hline & $\mathbf{( k c a l / m o l )}$ & $\mathbf{( k c a l} / \mathbf{m o l})$ & $\mathbf{( k c a l / m o l )}$ & $\mathbf{( k c a l} / \mathbf{m o l})$ & $\mathbf{( k c a l} / \mathbf{m o l})$ \\
\hline LigHAO-2YB91 & $-48,13 \pm 16,22$ & $-157,58 \pm 26,34$ & $-165,39 \pm 15,75$ & $296,13 \pm 19,68$ & $-21,27 \pm 1,71$ \\
Lig783-2YB9 & $-44,03 \pm 12,05$ & $-14,95 \pm 10,04$ & $-89,35 \pm 11,43$ & $72,10 \pm 25,33$ & $-11,83 \pm 1,26$ \\
Lig1022-2YB9 & $-66,32 \pm 16,72$ & $0,86 \pm 12,31$ & $-129,68 \pm 17,31$ & $78,69 \pm 25,78$ & $-16,19 \pm 2,22$ \\
Lig2177-2YB9 & $-65,64 \pm 30,36$ & $-106,32 \pm 9,38$ & $-203,96 \pm 10,13$ & $265,90 \pm 24,95$ & $-21,25 \pm 0,80$ \\
Lig3444-2YB9 & $78,99 \pm 18,67$ & $-38,94 \pm 23,86$ & $-114,74 \pm 11,73$ & $88,19 \pm 20,43$ & $-13,45 \pm 1,27$ \\
Lig6199-2YB9 & $-71,63 \pm 15,62$ & $-44,68 \pm 19,86$ & $-127,44 \pm 12,48$ & $116,52 \pm 22,91$ & $-16,03 \pm 1,35$ \\
\hline
\end{tabular}

${ }^{1}$ Ligand of reference from Protein Data Bank $(29,39)$

When analyzing the free energy decomposition presented in Table 2, we can observe that the electrostatic component, the Van der Waals interactions, and the non-polar solvation term were the stabilizing contributions of the systems studied except for the Lid1022-2YB9 complex. In this case, the electrostatic contribution was destabilizing. Considering that $\mathrm{H}$-bond is a type of electrostatic interaction, it is necessary to emphasize that this complex was the one with the less hydrogen bond numbers in the molecular dynamics simulations and low stability with an occupancy of less than $0.54 \%$ in all cases analyzed. This fact could be the explanation for the destabilizing positive value of $\Delta$ Eelect.

From Table 2, we can also observe that the Van der Waals term contributes the most to the complexes stability studied, with the most negative energy of all the energy contributions calculated using the MM-PBSA method. Let's analyze the ligands' structure in this work (Figure 8) and the docking experiments' results (Figure 2). We can observe that the non-polar hydrocarbon skeletons present attractive hydrophobic interactions with different amino acids in the NEP's active center. This approach agrees with the non-polar solvation term results, which also contributed positively to the stability of the complexes studied.

\subsection{Ligand Efficiency metrics and ADME-Tox Properties.}

One of the principal objectives of this work is to analyze which of the possible antihypertensive agents is the best candidate, minimizing the risk as much as possible. According to this goal, we have performed ligand efficiency calculations and in silico predictions of the pharmacokinetic (ADME) and toxicological (Tox) properties, considering the Lipinski $(40,41)$, Veber (42) , and Pfizer 3/75 (43) empirical rules.

Table 3 shows the behavior of these parameters for each molecule studied. There were significant differences in the $\mathrm{Kd}$ parameter (dissociation constant) of our reference ligand (LigHAO) for the compounds designed in silico with the lowest $\mathrm{Kd}$ value of all the molecules studied. The lower Kd value indicates a strong interaction between the ligand and the protein.

Of the in silico designed ligands, Lig6199 and Lig1022 had the lowest Kd values, indicating the strong interaction with Neprilysin. These results agree with the free energy calculations by the MM-PBSA method, resulting in these two ligands with the second and the third most negative binding energy of all complexes analyzed.

The ligand efficiency index (LE) is a parameter that correlates the binding energy obtained from docking experiments and the number of the atoms of the ligand, without considering the hydrogen atoms (44). Several researchers have suggested that for a molecule to be a good drug candidate, the LE value must be greater than $0.3 \mathrm{kcal} / \mathrm{mol}$ (44-47). So in this work, we take this value as a reference. 
Table 3. Ligand efficiency calculation of the firsts ranked docking poses complexes and ADME molecular descriptors of all compounds designed in silico to inhibit the Neutral Endopeptidase.

\begin{tabular}{lcccccc}
\hline \multirow{2}{*}{ Properties } & \multicolumn{5}{c}{ Ligand Efficiency Calculation } \\
\cline { 2 - 7 } & LigHAO & Lig783 & Lig1022 & Lig2177 & Lig3444 & Lig6199 \\
\hline Kd & $5,5 \times 10^{-16}$ & $6,05 \times 10^{-6}$ & $7,27 \times 10^{-8}$ & $6,86 \times 10^{-7}$ & $7,72 \times 10^{-7}$ & $3,39 \times 10^{-8}$ \\
LE & 0,6519 & 0,3560 & 0,2952 & 0,2474 & 0,3791 & 0,2732 \\
BEI & 34,54 & 19,15 & 15,83 & 12,82 & 17,16 & 14,51 \\
LLE & 11,63 & 2,61 & 1,27 & 2,71 & 3,80 & 3,34 \\
\hline & & ADME-Tox Properties. & & \\
\hline MW (Da) & 442,50 & 272,38 & 450,70 & 480,58 & 356,17 & 510,62 \\
cLogP & 2,84 & 2,60 & 5,86 & 3,45 & 2,31 & 4,06 \\
HBA & 7 & 2 & 2 & 5 & 3 & 7 \\
HBD & 3 & 2 & 0 & 2 & 5 & 1 \\
RB & 12 & 0 & 14 & 8 & 2 & 13 \\
TPSA $\left(\AA^{2}\right)$ & 129,73 & 40,46 & 31,14 & 114,67 & 76,55 & 88,54 \\
\hline
\end{tabular}

MW: Molecular weight; LogP: Octanol/water partition coefficient; HBA: Hydrogen bond acceptor; HBD: Hydrogen bond donor; TPSA: Topological polar surface area; RB: Rotatable bond count.

Considering the results shown in Table 3, our reference ligand (LigHAO) had the highest LE value $(0.6519 \mathrm{kcal} / \mathrm{mol})$; however, of the designed ligands, only Lig3444 and Lig783 had values somewhat higher than the reference value $(0.3791$ and $0.3560 \mathrm{kcal} / \mathrm{mol}$ respectively). These two molecules could be good candidates for anti-hypertensive agents; however, it is necessary to analyze other parameters to reinforce or reject this hypothesis.

The binding Efficiency Index (BEI) parameter relates to the ligand'sing energy of the thP and the ligand's molecular weight $(48,49)$. We have taken EIB values higher than 20 and lower than 27 as a reference value base on some drugs in the market like Bortezomid $(B E I=21)(50)$, with EIB values in this range. As shown in Table 3, none of the molecules studied had BEI values within this range. The ligands designed in silico had values below the reference range. They could be due to these molecules' high molecular weight, which could be a negative aspect in the design of new anti-hypertensive drugs.

Another important parameter is the Lipophilic Ligand Efficiency (LLE). This aspect relates to the binding energy obtained from docking experiments with the lipophilic power (CITA). As a reference value, we consider LLE numbers between 5 and 7 units (QUOTE). It is noteworthy that the behavior of this variable was similar to BEI. None of the molecules designed in silico had LLE values in the reference range, indicating that the compounds studied have high lipophilic power, a negative behavior for a drug candidate since it can accumulate in adipose tissue and cause repeated dose toxicity.

To corroborate the above, we performed pharmacokinetic (absorption, distribution, metabolism, and elimination (ADME)) and toxicological (Tox) predictions through calculations of different parameters, shown in Table 3. As a toxicological criterion, we compared the 
toxicological predictions with the empirical rules of Lipinski(40,41), Veber(42), and Pfizer 3/75(43) (Table 4 and 5).

Table 4. Verification of the empirical rules for the prediction of Bio-availability and Toxicity of the compounds designed in silico: Red represents the violation of any of the empirical rules, and blue the No violation of any empirical rule.

\begin{tabular}{|c|c|c|c|c|c|c|c|c|c|c|c|c|c|c|c|c|c|c|}
\hline \multirow{2}{*}{ Properties } & \multicolumn{3}{|c|}{ LigHAO } & \multicolumn{3}{|c|}{ Lig783 } & \multicolumn{3}{|c|}{ Lig1022 } & \multicolumn{3}{|c|}{ Lig2177 } & \multicolumn{3}{|c|}{ Lig3444 } & \multicolumn{3}{|c|}{ Lig6199 } \\
\hline & LR & VR & PR & LR & VR & PR & LR & VR & PR & LR & VR & PR & LR & VR & PR & LR & VR & PR \\
\hline MW (Da) & & - & - & & - & - & & - & & & - & - & & - & - & & - & - \\
\hline cLogP & & - & & & - & & & - & & & - & & & - & & & - & \\
\hline HBA & & - & - & & - & - & & - & - & & - & - & & - & - & & - & - \\
\hline HBD & & - & - & & - & - & & - & - & & - & - & & - & - & & - & - \\
\hline TPSA $\left(\AA^{2}\right)$ & - & & & - & & & - & & & - & & & - & & & - & & \\
\hline ER & - & & - & - & & - & - & & - & - & & - & - & & - & - & & - \\
\hline
\end{tabular}

LR: Lipinski Rules (40,41); VR: Veber Rules (42); PR: Pfizer 3/75 Rules (43)

According to the results shown in Table 3 and which were integrated into Table 4, we can observe that our reference ligand meets all the criteria contemplated in the Lipinski rule. However, it does not meet the rotatable bonds criteria in Veber's rule or the TPSA (Topological Polar Surface Area) criteria from Pfizer's rule. These results indicate that LigHAO is a very flexible molecule and exceeds the polarity range, so we recommend performing experimental tests if this compound is understood as a possible anti-hypertensive compound to know if there is a toxicity mechanism.

Of all the compounds designed in silico, Lig783 was the only one that meets all the parameters contemplated in the empirical rules of Lipinski, Veber, and Pfizer, which could be the right candidate for an anti-hypertensive agent. The other ligand to consider is Lig3444, which complies with all the Lipinski and Veber rule parameters; however, like our reference ligand, it does not comply with the TPSA parameter of the Pfizer Rule. Therefore, it is necessary to be not conclusive with this compound without doing experiments to evaluate the possible toxicity mechanism.

\section{Computational Details}

As shown previously, our group has designed a series of ligands as possible anti-hypertensive agents $(18,24)$, so the fundamental objective of this work is to analyze whether these designed compounds will be good candidates, taking Neutral Endopeptidase (NEP) as the target protein. To this, we have developed a sequential computational protocol, which is shown in Figure 7.

The computational protocol designed is presented in Figure 7 and will allow us to perform a comprehensive analysis of each studied molecule. The best candidates for anti-hypertensive agents will emerge from this analysis. This protocol will be explained in detail below. 


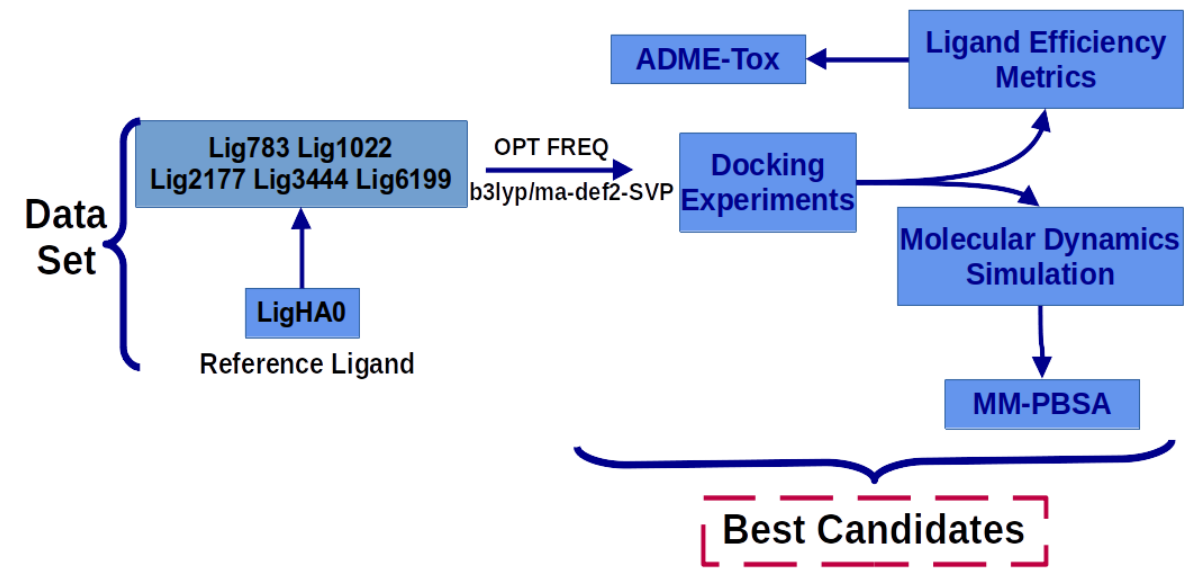

Figure 7. Sequential computational protocol for the evaluation of possible anti-hypertensive agents designed in silico.

\subsection{Data Set.}

We have designed several compounds as possible anti-hypertensive agents in previous work using the QSAR-IN and virtual screening methods (18). This result is the starting point to evaluate if any of these ligands could have this property. All molecules are represented in Figure 8, sketched using Avogadro software version 1.2.0(51).

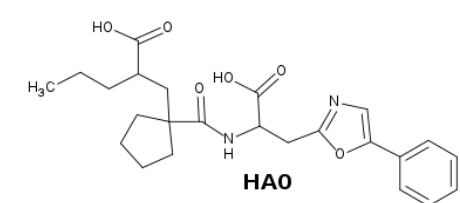

HETEROARYLALANINE 5-PHENYL OXAZOLE
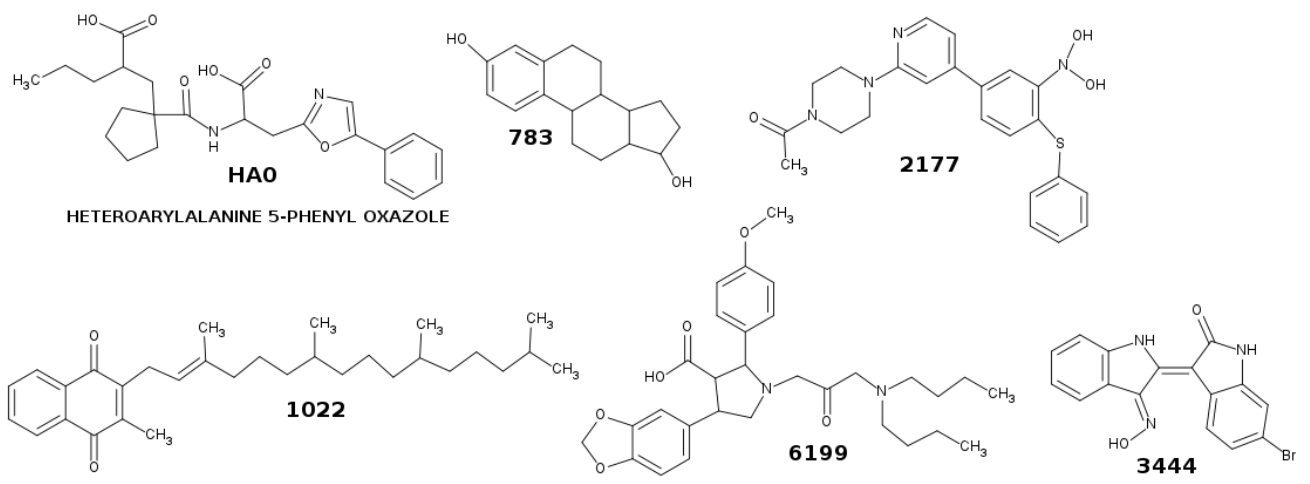

Figure 8. 2D molecular structure of NEP inhibitors as possible anti-hypertensive ligands.

The optimized geometry was obtained by DFT calculation at the b3lyp/ma-def2-SVP basis set implemented in Orca 4.2.1 software package(52,53). The full optimized geometry was checked by counting their imaginary frequencies for each ligand. The fully optimized geometries of the molecules (Figure 8) were obtained using docking experiments to examine the compounds' interactions in the Neprilysin (NEP) pocket.

\subsection{Docking Experiments.}

The optimized geometry (from the quantum calculation of the ligands) were used for docking experiments. The compounds were prepared at $\mathrm{pH}=7.4$ using Autodock Tools(54). The NEP X-ray crystallography structure was obtained from Protein Data Bank (PDB)(29), whose PDB id is 2 YB9, resolved at $2.40 \AA(39)$. This protein was prepared by the addition of all hydrogen atoms at $\mathrm{pH}=7.4$. The water molecules around the protein were eliminated, except those at a distance less than $5 \AA$ from our reference ligand LigHAO. The grid box's size was $25 \times 25 \times 25 \AA^{3}$ around the mass centers of the LigHAO (Heteroaryl-alanine-5-phenyl oxazole). Our reference ligands discharged from $x$-ray crystallography structure from PDB. The grid coordinates were $x=31,959 y=-43,612$ and 
$z=37,509$ and the cluster size was 8 . Neutral Endopeptidase is a metalloprotein that contains zinc $\left(\mathrm{Zn}^{2+}\right)$ in its active center. This fact was maintained in all docking experiments.

All docking experiments were performed under an accurate model with the flexibility of any amino acid side chain within $3 \AA$ of the ligand. All docking was realized with the SwissDock web server $(55,56)$. To analyze if our docking results were correct, the LigHAO reference ligand was re-docked using the same docking protocol of the other compounds. The best docking poses were selected using binding energy ( $\mathrm{kcal} / \mathrm{mol})$, FullFitness $(\mathrm{kcal} / \mathrm{mol})(56)$, and the positional root-meansquare deviation (RMSD)(27).

The best energetically favorable poses, the highest value of full fitness, and lowest root-meansquare deviation of each complex were selected for molecular dynamics simulations, MM-PBSA, and ligand efficiency calculations. To verify the docking results reproducibility, we calculate the root-mean-square deviation (RMSD) between the ligand designed in silico, and LigHAO as ligand reference from the Protein Data Bank crystallographic structure. These calculations were performed using the LigRMSD server 1.0 program (57). All docking figures were built using Pymol software version $1.8(58)$.

\subsection{Molecular Dynamics Simulation.}

We obtained the best conformational poses for each ligand-NEP complex as input for molecular dynamics simulations from docking experiments. Each complex was placed into a water box of 20x20x20 ̊ using the TIP3P water model $(59,60)$. Topologies and parameters designed in silico were obtained by the SwissParam Web Server (61). All molecular dynamics simulations were described using CHARMM36 and CGenFF force field for the Neutral Endopeptidase and the possible anti-hypertensive compounds (62-67).

The ligands-NEP complexes were submitted to 50000 steps for the energy minimization using the conjugated gradient methodology, reducing any close contact. The working temperature was $298.15 \mathrm{~K}$ employing the weak coupling algorithm (68). The Van der Waals cutoff was fixed to $12 \AA$, and we applied a backbone constraint to all complexes using the NPT ensemble. The Particle Mesh Ewald (PME) approach (69) was used for considering the long ranges of electrostatic forces. We used the velocity Verlet algorithm with a $1.0 \mathrm{fs}$ time step to solve the motion equations. All the complexes were submitted to $2.0 \mathrm{~ns}$ of equilibration and $50 \mathrm{~ns}$ of molecular dynamics simulation using the NAMD 2.13 software package (70). The trajectories analysis and scripts were perform using the VMD software version 1.9.3 (71).

\subsection{Free Energy Calculation by means MM-PBSA methods.}

This work's sequential computational protocol combines docking, molecular dynamics simulation, and MM-PBSA to study the ligands designed in silico interaction with Neutral Endopeptidase. The binding free energy was calculated using g_mmpbsa package version 5.1.2 (72), a Gromacs tool (73) to compute the ligand-NEP free binding energy. From 50 ns of molecular dynamics simulation, we extract the last 2500 frames to compute each complex's binding free energy. So the MM-PBSA method calculates the free energy decomposition into contributions. The free energy for the NEP-ligand complexes were calculated according to the following equation:

$$
\Delta G_{\text {binding }}=G_{\text {complex }}-\left(G_{N E P}+G_{\text {ligand }}\right)
$$

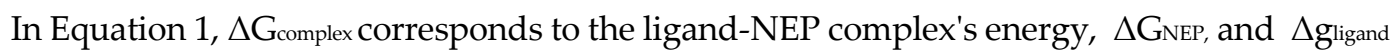
as protein and ligand energy, respectively. The following equation was used to calculate the protein's free energy, ligand, and complex separately. 


$$
G_{x}=E_{\text {bond }}+E_{v d w}+E_{\text {elect }}+G_{\text {polar }}+G_{\text {apolar }}
$$

In Equation 2, Gx can be G complex or GNEP, or Gligand. The Ebond represents the interactions that include bond, angle, and dihedral angle, Eelect is the electrostatic energy contribution, and Evdw is a Van der Waals energy contribution. The Gpolar represents the polar free energy contribution, which was calculated using the continuum solvent Poisson-Boltzmann (PB) model included in APBS (Adaptive Poisson-Boltzmann Solver) software version 1.4.1 (74). The non-polar free energy contribution was calculated according to the following equation:

$$
G_{\text {apolar }}=\gamma S A S A+\beta
$$

In Equation $3 \gamma$ represent the coefficient related to the solvent surface tension, which, in this work, was $0.0072 \mathrm{kcal} / \mathrm{mol} / \AA^{2}$, SASA represents the solvent-accessible surface area, with an amount of $1.4 \AA$, and $\beta$ is a fitting parameter. We also decompose the overall binding energy per residue because we need to know every amino acid's contribution. These contributions were calculated using pythons script MmPbSaStat.py(72).

\subsection{Ligand Efficiency Calculation.}

Ligand efficiency metrics consist of a series of parameters that we can use to measure the relationship between the binding energy and the molecule size (47). These parameters significantly predict how efficient a compound will be as a possible drug $(44,45,47,75)$. In this work, the ligand efficiency calculations were performed through several parameters shown in Table 5.

\begin{tabular}{|c|c|c|c|}
\hline Parameter & Description & Equation & Reference \\
\hline $\mathrm{Kd}$ & $\begin{array}{l}\text { Dissociation constant. It is value indicates the } \\
\text { bond strength between the ligand and the } \\
\text { protein. According to this Equation } 4 \Delta \mathrm{G} \text { docking, } \\
\text { the binding energy ( } \mathrm{kcal} / \mathrm{mol} \text { ) obtained from } \\
\text { docking experiments, } \mathrm{R} \text { is the gas constant } \\
\text { whose value is } 0,001987 \mathrm{kcal} / \mathrm{molK} \text {, and T is the } \\
\text { temperature in Kelvin, in our case, } 298.15 \mathrm{~K} \text {. }\end{array}$ & $K_{d}=10^{\left[\frac{\Delta G_{\text {docking }}}{2,303 R T}\right]}(4)$ & $(44,45)$ \\
\hline LE & $\begin{array}{l}\text { Ligand efficiency index. This parameter is a } \\
\text { measure of the binding energy and the size of } \\
\text { the compound. }\end{array}$ & $L E=\frac{-2,303 R T}{H A C} \log \left(K_{d}\right)(5)$ & $(44,45)$ \\
\hline BEI & $\begin{array}{l}\text { The Binding efficiency index (BEI) is a measure } \\
\text { that involves a ligand's binding property with } \\
\text { the protein against molecular weight. In } \\
\text { Equation } 6 \mathrm{pKd} \text { is -logKd, and } \mathrm{Kd} \text { is the } \\
\text { dissociation constant calculated from Equation } \\
\text { 4. MW represents the molecular weight in } \mathrm{kDa} \text {. }\end{array}$ & $B E I=\left[p K_{d} M W\right](6)$ & $(49,76)$ \\
\hline LLE & $\begin{array}{l}\text { LLE correspond to Lipophilic ligand efficiency } \\
\text { has been defined as the difference between the } \\
\text { ligand activity and lipophilicity }(\log P)\end{array}$ & $L L E=p K_{d}-\operatorname{clog}(P)(7)$ & (77) \\
\hline
\end{tabular}

Table 5. Parameters used for Ligand Efficiency Calculation: 


\subsection{ADME-Tox Properties.}

The absorption, distribution, metabolism, and excretion (ADME) properties of all ligands designed in silico were calculated from the full optimized geometry using the SwissADME web server (78). Also, we computed other physicochemical properties. Such as molecular weight (MW), octanol/water partition coefficient (cLogP), hydrogen bond acceptor (HBA), hydrogen bond donor (HBD), topological polar surface area (TPSA), and rotatable bond count (RB) respectively using SwissADME web server (78). Base on the physicochemical parameters, we can predict the toxicological properties (Tox) of our ligands. They were taking into account the Lipinski(40,41), Veber(42), and Pfizer 3/75 toxicity empirical rules(43) (Table 6).

Table 6. Empirical rules for predicting oral availability and toxicity properties of ligands studied.

\begin{tabular}{lccc}
\hline \multirow{2}{*}{ Properties } & \multicolumn{2}{c}{ Oral Availability } & Toxicity \\
\cline { 2 - 4 } & Lipinski Rules & Veber Rules & Pfizer 3/75 Rules \\
\hline MW & $\leq 500$ & - & - \\
cLogP & $\leq 5$ & - & $\leq 3$ \\
HBA & $\leq 10$ & - & - \\
HBD & $\leq 5$ & - & - \\
TPSA & - & $\leq 140$ & $\leq 75$ \\
RB & - & $\leq 10$ & - \\
\hline
\end{tabular}

MW: Molecular weight; LogP: Octanol/water partition coefficient; HBA: Hydrogen bond acceptor; HBD: Hydrogen bond donor; TPSA: Topological polar surface area; RB: Rotatable bond count.

\section{Conclusions}

Arterial hypertension is one of the health problems that most affect the population worldwide. Given this disease's etiology, hypertensive patients are almost forced to increase the drug dose or change it. Many researchers have been working on designing possible anti-hypertensive agents more effective and reducing as much as possible the side effects that some drugs present on the market. How we know if the compounds designed could be good anti-hypertensive agents?. To answer this question, we successfully applied a sequential computational protocol that allowed us, through a comprehensive analysis of the results, to select which compounds previously designed in silico by our group could be good anti-hypertensive agents.

The top results we have obtained were that the ligands were oriented adequately in the NEP's active center compared to our reference ligand using the docking experiments. However, we lost interactions because of hydrogen bonding from the molecular dynamics analysis, where LIg783 and Lig3444 formed the most stable complexes. This result agrees with those obtained in the calculations of free binding energy using the MM-GBSA Method in which the complex formed by Lig3444-2YB9 was with the most negative binding energy. These two ligands could be considered good candidates for anti-hypertensive agents based on the ADME-Tox predictions' favorable results. However, this result is not conclusive, first, it is necessary to perform other experimental tests that support our result.

Author Contributions: K.M.U and EL. Conceived and designed the study. J.C.C. conceived and realized the docking experiments and Molecular Dynamics Simulation, J.C.G realized the MM-PBSA calculation, E.L. performed the Ligand Efficiency Calculation, and drafted the first version of the paper, K.M.U., conceived and 
realized the ADME-Tox properties and contributed to the revision process of the manuscript and submit the paper. All authors reviewed the manuscript.

Funding: This work was funded by FONDECYT Iniciación grant $N^{\circ} 11180650$.

Conflicts of Interest: The authors declare no conflict of interest.

\section{Abbreviations}

RAAS Renin-Angiotensin-Aldosterone System

NEP Neutral Endopeptidase (Neprilisin)

RMSD Root Means Squared Deviation

RMSF Root Means Squared Fluctuation

MD Molecular Dynamics Simulation

LE Ligand Efficiency Index

BEI Binding Efficiency Index

LEE Lipophilic Ligand Efficiency

\section{References}

1. WHO | Raised blood pressure. WHO [Internet]. 2016 [cited 2017 Apr 2]; Available from: http://www.who.int/gho/ncd/risk_factors/blood_pressure_text/en/

2. Petermann F, Durán E, Labraña AM, Martínez MA, Leiva AM, Garrido A, et al. Risk factors associated with hypertension. Analysis of the 2009-2010 Chilean health survey. Rev Med Chil [Internet]. 2017;145(8):996-1004. Available from: https:/scielo.conicyt.cl/pdf/rmc/v145n8/0034-9887-rmc-145-080996.pdf

3. Muñoz-Durango N, Fuentes CA, Castillo AE, González-Gómez LM, Vecchiola A, Fardella CE, et al. Role of the renin-angiotensin-aldosterone system beyond blood pressure regulation: Molecular and cellular mechanisms involved in end-organ damage during arterial hypertension. Int J Mol Sci. 2016;17(7):1-17.

4. Te Riet L, Van Esch JHM, Roks AJM, Van Den Meiracker AH, Danser AHJ. Hypertension: ReninAngiotensin-Aldosterone System Alterations. Circ Res. 2015;116(6):960-75.

5. Hubers SA, Brown NJ. Combined Angiotensin Receptor Antagonism and Neprilysin Inhibition. Circulation [Internet]. 2016 [cited 2017 Apr 2];133(11). Available from: http://circ.ahajournals.org/content/133/11/1115.short

6. Patten GS, Abeywardena MY, Bennett LE. Inhibition of Angiotensin Converting Enzyme, Angiotensin II Receptor Blocking, and Blood Pressure Lowering Bioactivity across Plant Families. Crit Rev Food Sci Nutr [Internet]. 2016 Jan 25 [cited 2017 Apr 2];56(2):181-214. Available from: http://www.tandfonline.com/doi/full/10.1080/10408398.2011.651176

7. Stanisz B, Regulska K, Regulski M. The angiotensin converting enzyme inhibitors - alternative clinical applications. J Med Sci [Internet]. 2016 [cited 2017 Apr 2];83(1):57-61. Available from: http://jms.ump.edu.pl/index.php/JMS/article/view/45 
8. Ruschitzka F, Corti R, Quaschning T, Hermann M, Lüscher TF. Vasopeptidase inhibitors--concepts and evidence. Nephrol Dial Transplant [Internet]. 2001 Aug 1 [cited 2017 Apr 3];16(8):1532-5. Available from: https://academic.oup.com/ndt/article-lookup/doi/10.1093/ndt/16.8.1532

9. Griggs DW, Prinsen MJ, Oliva J, Campbell MA, Arnett SD, Tajfirouz D, et al. Pharmacologic Comparison of Clinical Neutral Endopeptidase Inhibitors in a Rat Model of Acute Secretory Diarrhea. J Pharmacol Exp Ther [Internet]. 2016 [cited 2017 Apr 3];357(2). Available from: http://jpet.aspetjournals.org/content/357/2/423.short

10. Daskaya-Dikmen C, Yucetepe A, Karbancioglu-Guler F, Daskaya H, Ozcelik B. Angiotensin-IConverting Enzyme (ACE)-Inhibitory Peptides from Plants. Nutrients [Internet]. 2017 Mar 23 [cited 2018 May 14];9(4):316. Available from: http://www.mdpi.com/2072-6643/9/4/316

11. McDowell G, Coutie W, Shaw C, Buchanan KD, Struthers AD, Nicholls DP. The neutral endopeptidase inhibitor drug, candoxatril, on circulating levels of two of the most potent vasoactive peptides. Br J Clin Pharmacol [Internet]. 2003 Nov 21 [cited 2017 Apr 3];43(3):329-32. Available from: http://doi.wiley.com/10.1046/j.1365-2125.1997.00545.x

12. Hyoung Lee D, Ho Kim J, Sik Park J, Jun Choi Y, Soo Lee J. Isolation and characterization of a novel angiotensin I-converting enzyme inhibitory peptide derived from the edible mushroom Tricholoma giganteum. Peptides [Internet]. 2004 [cited 2017 Apr 3];25(4):621-7. Available from: http://www.sciencedirect.com/science/article/pii/S0196978104001196

13. Kataoka H, Hayatsu T, Hietsch G, Steinkellner H, Nishioka S, Narimatsu S, et al. Identification of mutagenic heterocyclic amines (IQ, Trp-P-1 and AalphaC) in the water of the Danube river. Mutat Res [Internet]. 2000 Mar 3;466(1):27-35. Available from: http://www.ncbi.nlm.nih.gov/pubmed/10751722

14. Corti R, Burnett Jr JC, Rouleau JL, Ruschitzka F, Lüscher TF. Vasopeptidase Inhibitors. Circulation [Internet]. 2001 [cited 2017 Apr 3];104(15). Available from: http://circ.ahajournals.org/content/104/15/1856

15. Kataria V, Wang H, Wald JW, Phan YL. Lisinopril-Induced Alopecia: A Case Report. J Pharm Pract [Internet]. 2017 Oct 6 [cited 2018 May 6];30(5):562-6. Available from: http://journals.sagepub.com/doi/10.1177/0897190016652554

16. Karimi-Maleh H, Ganjali MR, Norouzi P, Bananezhad A. Amplified nanostructure electrochemical sensor for simultaneous determination of captopril, acetaminophen, tyrosine and hydrochlorothiazide. Mater Sci Eng C [Internet]. 2017 Apr 1 [cited 2018 May 6];73:472-7. Available from: https://www.sciencedirect.com/science/article/pii/S0928493116320367 
17. Bhat ZF, Kumar S, Bhat HF. Anti-hypertensive peptides of animal origin: A review. Crit Rev Food Sci Nutr [Internet]. 2017 Feb 11 [cited 2018 May 6];57(3):566-78. Available from: https://www.tandfonline.com/doi/full/10.1080/10408398.2014.898241

18. Cañizares-Carmenate Y, Mena-Ulecia K, Perera-Sardiña Y, Torrens F, Castillo-Garit JA. An approach to identify new anti-hypertensive agents using Thermolysin as model: In silico study based on QSARINS and docking. Arab J Chem. 2019;12(8):4861-77.

19. Holland DR, Matthews BW, Barclay PL, Danilewicz JC, James K. Inhibition of Thermolysin and Neutral Endopeptidase 24.11 by a Novel Glutaramide Derivative: X-ray Structure Determination of the Thermolysin-Inhibitor Complex. Biochemistry [Internet]. 1994 Jan 1 [cited 2020 Nov 17];33(1):51-6. Available from: https://pubmed.ncbi.nlm.nih.gov/8286362/

20. Ren L, Lu X, Danser AHJ. Revisiting the Brain Renin-Angiotensin System-Focus on Novel Therapies. Curr Hypertens Rep [Internet]. 2019 Apr 4 [cited 2019 Nov 7];21(4):28. Available from: http://link.springer.com/10.1007/s11906-019-0937-8

21. Adekoya OA, Sylte I. The Thermolysin Family (M4) of Enzymes: Therapeutic and Biotechnological Potential. Chem Biol Drug Des [Internet]. 2009 Jan [cited 2019 Oct 25];73(1):7-16. Available from: http://www.ncbi.nlm.nih.gov/pubmed/19152630

22. Nehme A, Zouein FA, Zayeri ZD, Zibara K. An Update on the Tissue Renin Angiotensin System and Its Role in Physiology Pathology. J Cardiovasc Dev Dis [Internet]. 2019 Mar 29 [cited 2019 Nov 7];6(2):14. Available from: https://www.mdpi.com/2308-3425/6/2/14

23. Quesada-Romero L, Mena-Ulecia K, Tiznado W, Caballero J. Insights into the interactions between maleimide derivates and GSK3 $\beta$ combining molecular docking and QSAR. PLoS One. 2014;9(7).

24. MacLeod-Carey D, Solis-Céspedes E, Lamazares E, Mena-Ulecia K. Evaluation of new anti-hypertensive drugs designed in silico using Thermolysin as a target. Saudi Pharm J. 2020 May 1;28(5):582-92.

25. Mena-Ulecia K, MacLeod-Carey D. Interactions of 2-phenyl-benzotriazole xenobiotic compounds with human Cytochrome P450-CYP1A1 using docking, molecular dynamics simulations and MM-GBSA calculations. Comput Biol Chem [Internet]. 2018;74:253-62. Available from: https://doi.org/10.1016/j.compbiolchem.2018.04.004

26. Glossop MS, Bazin RJ, Dack KN, Fox DNA, Macdonald GA, Mills M, et al. Synthesis and Evaluation of Heteroarylalanine Diacids as Potent and Selective Neutral Endopeptidase Inhibitors. BioorgMedChemLett [Internet]. 2011 [cited 2018 May 7];21:3404. Available from: https://www.rcsb.org/structure/2YB9 
27. Gohlke H, Hendlich M, Klebe G. Knowledge-based scoring function to predict protein-ligand interactions. J Mol Biol [Internet]. 2000 Jan 14 [cited 2016 Feb 20];295(2):337-56. Available from: http://www.sciencedirect.com/science/article/pii/S0022283699933715

28. Kitchen DB, Decornez H, Furr JR, Bajorath J. Docking and scoring in virtual screening for drug discovery: methods and applications. Nat Rev Drug Discov [Internet]. 2004 Nov [cited 2013 May 22];3(11):935-49. Available from: http://www.ncbi.nlm.nih.gov/pubmed/15520816

29. Berman H. M, Westbrook J, Feng Z, Gilliland G, Bhat TN, Weissig H, et al. The Protein Data Bank. Nucleic Acids Res [Internet]. 2000 Jan 1 [cited 2017 Aug 7];28(1):235-42. Available from: http://www.ncbi.nlm.nih.gov/pubmed/10592235

30. Kumar A, Purohit R. Use of Long Term Molecular Dynamics Simulation in Predicting Cancer Associated SNPs. PLoS Comput Biol. 2014;10(4).

31. Lavanya P, Ramaiah S, Anbarasu A. A Molecular Docking and Dynamics Study to Screen Potent AntiStaphylococcal Compounds Against Ceftaroline Resistant MRSA. J Cell Biochem. 2016;117(2):542-8.

32. Kumar KM, Anbarasu A, Ramaiah S. Molecular docking and molecular dynamics studies on $\beta$ lactamases and penicillin binding proteins. Mol Biosyst [Internet]. 2014;10(4):891-900. Available from: http://www.ncbi.nlm.nih.gov/pubmed/24503740

33. Mohammad A, Marafie SK, Alshawaf E, Abu-Farha M, Abubaker J, Al-Mulla F. Structural analysis of ACE2 variant N720D demonstrates a higher binding affinity to TMPRSS2. Life Sci. 2020 Oct 15;259:118219.

34. Abroshan H, Akbarzadeh H, Parsafar GA. Molecular dynamics simulation and MM-PBSA calculations of sickle cell hemoglobin in dimer form with Val, Trp, or Phe at the lateral contact. J Phys Org Chem [Internet]. 2010 Sep 16 [cited 2013 Oct 12];23(9):866-77. Available from: http://doi.wiley.com/10.1002/poc.1679

35. El-Barghouthi MII, Jaime C, Al-Sakhen N a., Issa a. a., Abdoh a. a., Al Omari MMM, et al. Molecular dynamics simulations and MM-PBSA calculations of the cyclodextrin inclusion complexes with 1alkanols, para-substituted phenols and substituted imidazoles. J Mol Struct THEOCHEM [Internet]. 2008 Mar [cited 2013 May 29];853(1-3):45-52. Available from: http://linkinghub.elsevier.com/retrieve/pii/S0166128007007749

36. Genheden S, Ryde U. The MM/PBSA and MM/GBSA methods to estimate ligand-binding affinities. Expert Opin Drug Discov. 2015;10(5):449-61.

37. Su P-C, Tsai C-C, Mehboob S, Hevener KE, Johnson ME. Comparison of radii sets, entropy, QM methods, and sampling on MM-PBSA, MM-GBSA, and QM/MM-GBSA ligand binding energies of $F$. tularensis 
enoyl-ACP reductase (FabI). J Comput Chem [Internet]. 2015 Sep 30 [cited 2017 May 10];36(25):1859-73. Available from: http://doi.wiley.com/10.1002/jcc.24011

38. Rai N, Muthukumaran R, Amutha R. Identification of inhibitor against H. pylori HtrA protease using structure-based virtual screening and molecular dynamics simulations approaches [Internet]. Vol. 118, Microbial Pathogenesis. Elsevier Ltd; 2018. 365-377 p. Available from: https://doi.org/10.1016/j.micpath.2018.03.027

39. Glossop MS, Bazin RJ, Dack KN, Fox DNA, MacDonald GA, Mills M, et al. Synthesis and evaluation of heteroarylalanine diacids potent and selective neutral endopeptidase inhibitors. Vol. 21, Bioorganic \& Medicinal Chemistry Letters. 2011.

40. Lipinski CA, Lombardo F, Dominy BW, Feeney PJ. Experimental and computational approaches to estimate solubility and permeability in drug discovery and development settings. Adv Drug Deliv Rev [Internet]. 2001 Mar 1 [cited 2019 Oct 21];46(1-3):3-26. Available from: http://www.ncbi.nlm.nih.gov/pubmed/11259830

41. Duchowics PR, Talevi A, Bellera C, Bruno-Blanch LE, Castro EA, Duchowicz PR, et al. Application of descriptors based on Lipinski's rules in the QSPR study of aqueous solubilities. Bioorg Med Chem [Internet]. 2007 Jun 1 [cited 2013 May 29];15(11):3711-9. Available from: http://www.ncbi.nlm.nih.gov/pubmed/17418580

42. Veber DF, Johnson SR, Cheng H-Y, Smith BR, Ward KW, Kopple KD. Molecular Properties That Influence the Oral Bioavailability of Drug Candidates. J Med Chem [Internet]. 2002 Jun 6 [cited 2019 Oct 21];45(12):2615-23. Available from: http://www.ncbi.nlm.nih.gov/pubmed/12036371

43. Hughes JD, Blagg J, Price DA, Bailey S, DeCrescenzo GA, Devraj R V., et al. Physiochemical drug properties associated with in vivo toxicological outcomes. Bioorg Med Chem Lett [Internet]. 2008 Sep 1 [cited 2019 Oct 21];18(17):4872-5. Available from: https://www.sciencedirect.com/science/article/pii/S0960894X08008500?via\%3Dihub

44. Abad-Zapatero C. Ligand Efficiency Indices for Drug Discovery. Ligand Effic Indices Drug Discov. 2013;10(7):469-88.

45. Abad-Zapatero C, Perišić O, Wass J, Bento AP, Overington J, Al-Lazikani B, et al. Ligand efficiency indices for an effective mapping of chemico-biological space: The concept of an atlas-like representation. Drug Discov Today. 2010;15(19-20):804-11.

46. Hopkins AL, Keserü GM, Leeson PD, Rees DC, Reynolds CH. The role of ligand efficiency metrics in drug discovery. Nat Rev Drug Discov. 2014 Feb;13(2):105-21.

47. Murray CW, Erlanson DA, Hopkins AL, Keserü GM, Leeson PD, Rees DC, et al. Validity of ligand efficiency metrics. ACS Med Chem Lett. 2014;5(6):616-8. 
48. Kenny PW. The nature of ligand efficiency. J Cheminform. 2019 Jan 31;11(1):1-18.

49. Kenny PW, Leitão A, Montanari CA. Ligand efficiency metrics considered harmful. Vol. 28, Journal of Computer-Aided Molecular Design. Kluwer Academic Publishers; 2014. p. 699-710.

50. Xu Y, Yang X, Chen Y, Chen H, Sun H, Li W, et al. Discovery of novel 20 S proteasome inhibitors by rational topology-based scaffold hopping bortezomib. Bioorganic Med Chem Lett. 2018 Jul 1;28(12):2148-52.

51. Hanwell MD, Curtis DE, Lonie DC, Vandermeersch T, Zurek E, Hutchison GR. Avogadro: an advanced semantic chemical editor, visualization, and analysis platform. J Cheminform [Internet]. 2012;4(1):17. Available from: https://doi.org/10.1186/1758-2946-4-17

52. Neese F. The ORCA program system. Wiley Interdiscip Rev Comput Mol Sci [Internet]. 2012 Jan [cited 2019 Oct 8];2(1):73-8. Available from: http://doi.wiley.com/10.1002/wcms.81

53. Neese F. Software update: the ORCA program system, version 4.0. Wiley Interdiscip Rev Comput Mol Sci [Internet]. 2018 Jan 1 [cited 2019 Oct 8];8(1):e1327. Available from: http://doi.wiley.com/10.1002/wcms.1327

54. Morris GM, Huey R, Lindstrom W, Sanner MF, Belew RK, Goodsell DS, et al. AutoDock4 and AutoDockTools4: Automated docking with selective receptor flexibility. J Comput Chem [Internet]. 2009 Dec [cited 2014 Oct 17];30(16):2785-91. Available from: http://www.pubmedcentral.nih.gov/articlerender.fcgi?artid=2760638\%7B\&\%7Dtool=pmcentrez\%7B\& \%7Drendertype=abstract

55. Grosdidier A, Zoete V, Michielin O. SwissDock, a protein-small molecule docking web service based on EADock DSS. Nucleic Acids Res [Internet]. 2011 Jul 1 [cited 2020 Nov 20];39(SUPPL. 2):W270-7. Available from: https://academic.oup.com/nar/article/39/suppl_2/W270/2506492

56. Bitencourt-Ferreira G, de Azevedo WF. Docking with SwissDock. In: Methods in Molecular Biology [Internet]. Humana Press Inc.; 2019 [cited 2020 Nov 20]. p. 189-202. Available from: https://link.springer.com/protocol/10.1007/978-1-4939-9752-7_12

57. Velázquez-Libera JL, Durán-Verdugo F, Valdés-Jiménez A, Núñez-Vivanco G, Caballero J. LigRMSD: a web server for automatic structure matching and RMSD calculations among identical and similar compounds in protein-ligand docking. Ponty Y, editor. Bioinformatics [Internet]. 2020 Jan 11 [cited 2020 Jan 27]; Available from: https://academic.oup.com/bioinformatics/advancearticle/doi/10.1093/bioinformatics/btaa018/5700716

58. Schrödinger, LLC. The \{PyMOL\} Molecular Graphics System, Version 1.3r1. 2010. 
59. Boonstra S, Onck PR, Giessen E van der. CHARMM TIP3P Water Model Suppresses Peptide Folding by Solvating the Unfolded State. J Phys Chem B [Internet]. 2016 Apr 21 [cited 2017 May 10];120(15):3692-8. Available from: http://pubs.acs.org/doi/abs/10.1021/acs.jpcb.6b01316

60. Lu J, Qiu Y, Baron R, Molinero V. Coarse-Graining of TIP4P/2005, TIP4P-Ew, SPC/E, and TIP3P to Monatomic Anisotropic Water Models Using Relative Entropy Minimization. J Chem Theory Comput [Internet]. 2014 Sep 9 [cited 2017 May 10];10(9):4104-20. Available from: http://pubs.acs.org/doi/abs/10.1021/ct500487h

61. Zoete V, Cuendet MA, Grosdidier A, Michielin O, Vincent Z, A. CM, et al. SwissParam: A fast force field generation tool for small organic molecules. J Comput Chem [Internet]. 2011 Aug [cited 2017 Feb 1];32(11):2359-68. Available from: http://doi.wiley.com/10.1002/jcc.21816

62. Lee J, Cheng X, Swails JM, Yeom MS, Eastman PK, Lemkul JA, et al. CHARMM-GUI Input Generator for NAMD, GROMACS, AMBER, OpenMM, and CHARMM/OpenMM Simulations Using the CHARMM36 Additive Force Field. J Chem Theory Comput [Internet]. 2016 Jan 12 [cited 2017 May 10];12(1):405-13. Available from: http://pubs.acs.org/doi/abs/10.1021/acs.jctc.5b00935

63. Brooks BR, Brooks CL, Mackerell AD, Nilsson L, Petrella RJ, Roux B, et al. CHARMM: The biomolecular simulation program. J Comput Chem [Internet]. 2009;30(10):1545-614. Available from: http://dx.doi.org/10.1002/jcc.21287

64. MacKerell AD, Feig M, Brooks CL. Improved treatment of the protein backbone in empirical force fields. J Am Chem Soc [Internet]. 2004 Jan 28;11. MacKer(3):698-9. Available from: http://www.ncbi.nlm.nih.gov/pubmed/14733527

65. Soteras Gutiérrez I, Lin F-Y, Vanommeslaeghe K, Lemkul JA, Armacost KA, Brooks CL, et al. Parametrization of halogen bonds in the CHARMM general force field: Improved treatment of ligandprotein interactions. Bioorg Med Chem [Internet]. 2016 [cited 2017 May 10];24(20):4812-25. Available from: http://www.sciencedirect.com/science/article/pii/S0968089616304576

66. Vanommeslaeghe K, MacKerell AD. CHARMM additive and polarizable force fields for biophysics and computer-aided drug design. Biochim Biophys Acta - Gen Subj [Internet]. 2015 May [cited 2017 May 10];1850(5):861-71. Available from: http://linkinghub.elsevier.com/retrieve/pii/S0304416514002736

67. Best RB, Zhu X, Shim J, Lopes PEM, Mittal J, Feig M, et al. Optimization of the additive CHARMM allatom protein force field targeting improved sampling of the backbone $\varphi, \psi$ and side-chain $\chi(1)$ and $\chi(2)$ dihedral angles. J Chem Theory Comput [Internet]. 2012 Sep 11;8(9):3257-73. Available from: http://www.ncbi.nlm.nih.gov/pubmed/23341755 
68. Berendsen HJC, Postma JPM, Gunsteren WF Van, Nola A Di, Haak JRK, van Gunsteren WF, et al. Molecular dynamics with coupling to an external bath. J Chem Physic [Internet]. 1984 [cited 2013 May 24];81(8):3684. Available from: http://link.aip.org/link/JCPSA6/v81/i8/p3684/s1\&Agg=doi

69. Onufriev A, Bashford D, Case DA. Exploring protein native states and large-scale conformational changes with a modified generalized born model. Proteins Struct Funct Bioinforma [Internet]. 2004;55(2):383-94. Available from: http://dx.doi.org/10.1002/prot.20033

70. Phillips JC, Braun R, Wang W, Gumbart J, Tajkhorshid E, Villa E, et al. Scalable molecular dynamics with NAMD. J Comput Chem [Internet]. 2005 Dec [cited 2013 May 21];26(16):1781-802. Available from: http://www.pubmedcentral.nih.gov/articlerender.fcgi?artid=2486339\%7B\&\%7Dtool=pmcentrez\%7B\& \%7Drendertype=abstract

71. Dalke W, Humphrey A, Schulten K, Humphrey W, Dalke A, Schulten K. VMD: visual molecular dynamics. J Mol Graph [Internet]. 1996 Mar;14(1):33-8. Available from: http://www.ncbi.nlm.nih.gov/pubmed/8744570

72. Kumari R, Kumar R, Lynn A. G-mmpbsa -A GROMACS tool for high-throughput MM-PBSA calculations. J Chem Inf Model. 2014 Jul 28;54(7):1951-62.

73. Abraham MJ, Murtola T, Schulz R, Páll S, Smith JC, Hess B, et al. GROMACS: High performance molecular simulations through multi-level parallelism from laptops to supercomputers. SoftwareX [Internet]. 2015 Sep 1 [cited 2020 May 7];1-2:19-25. Available from: https://linkinghub.elsevier.com/retrieve/pii/S2352711015000059

74. Baker NA, Sept D, Joseph S, Holst MJ, McCammon JA. Electrostatics of nanosystems: Application to microtubules and the ribosome. Proc Natl Acad Sci U S A [Internet]. 2001 Aug 28 [cited 2020 Nov 20];98(18):10037-41. Available from: www.pnas.orgcgidoi10.1073pnas.181342398

75. Meneses L, Cuesta S. Determinación Computacional de la Afinidad y Eficiencia de Enlace de Antinflamatorios No Esteroideos Inhibidores de la Ciclooxigenasa-2. Rev Ecuat Med Cienc Biol. 2017;36(1-2):17.

76. Polanski J, Tkocz A, Kucia U. Beware of ligand efficiency (LE): Understanding LE data in modeling structure-activity and structure-economy relationships. J Cheminform [Internet]. 2017 Sep 11 [cited 2020 May 11];9(1):49. Available from: https://jcheminf.biomedcentral.com/articles/10.1186/s13321-017-0236-9

77. Hopkins AL, Keserü GM, Leeson PD, Rees DC, Reynolds CH. The role of ligand efficiency metrics in drug discovery. Nat Rev Drug Discov. 2014 Feb;13(2):105-21.

78. Daina A, Michielin O, Zoete V. SwissADME: a free web tool to evaluate pharmacokinetics, druglikeness and medicinal chemistry friendliness of small molecules. Sci Rep [Internet]. 2017 May 3 [cited 2019 Oct 21];7(1):42717. Available from: http://www.ncbi.nlm.nih.gov/pubmed/28256516 
